\title{
Impacto do Diabetes Mellitus na Mortalidade em Síndromes Coronarianas Agudas
}

\begin{abstract}
RESUMO
O diabetes mellitus (DM) é uma das grandes causas de morte no mundo, principalmente em decorrência das doenças cardiovasculares. Atualmente, sabe-se que não somente o DM, como também os demais estados hiperglicêmicos, determinam um risco aumentado de doença arterial coronariana. No contexto das síndromes coronarianas agudas (SCA), o DM determina um pior prognóstico, tanto a curto quanto a longo prazo. Sendo o risco absoluto de mortalidade maior em diabéticos, as intervenções nessa população trazem maior impacto quanto aos benefícios. Estudos têm comprovado um maior benefício em diabéticos contra não-diabéticos na adoção de medidas como controle rigoroso da hiperglicemia intra-hospitalar, terapia de reperfusão (seja por trombólise, seja por intervenção percutânea), uso de inibidores da glicoproteína Ilb/llla e de inibidores da enzima conversora da angiotensina (ECA). Apesar dos benefícios da adoção de intervenções baseadas em evidências no tratamento das SCA em diabéticos, chama atenção a sub-utilização dessas medidas. Tendo em vista o aumento da prevalência do diabetes mellitus previsto para os próximos anos e levando-se em conta que as síndromes coronarianas agudas serão a principal causa de mortalidade nessa população, torna-se cada vez mais necessário que cardiologistas e endocrinologistas passem a interagir, de maneira a modificar o panorama previsto. (Arq Bras Endocrinol Metab 2007;51/2:275-280)
\end{abstract}

Descritores: Doenças cardiovasculares; Diabetes mellitus; Mortalidade; Medicina baseada em evidências

\begin{abstract}
The Impact of Diabetes Mellitus on the Mortality of Acute Coronary Syndromes.

Diabetes mellitus (DM) is a leading cause of mortality in the world, mainly on account of cardiovascular diseases. At present we know that not only DM but also other hyperglycemic states are a risk factor for coronary arterial disease. In the context of acute coronary syndromes, DM determines a worst prognosis, either in short- or long-term outcomes. Since the absolute risk of death is greater among diabetic patients when compared to non-diabetic patients, therapeutical interventions have a greater impact in terms of benefits to these patients as well. Strategies such as strict control of hyperglycemia during hospitalization, acute reperfusion management (either by thrombolysis or by percutaneous coronary intervention), use of platelet glycoprotein Ilb/llla inhibitors and angiotensin-converting enzyme (ACE)-inhibitors have recently proven to be of greater benefit for diabetics over non-diabetic patients. Meanwhile, in spite of all proven benefits of the use of evidence-based interventions to the treatment of acute coronary syndromes on diabetic patients, there is still an under utilization of these measures. Therefore, taking into account the predictions of an increasing number of diabetics in the world for the future years, and the fact that acute coronary syndromes will be the leading cause of death among them, it becomes increasingly necessary for both cardiologists and endocrinologists to work together in order to reduce the unfavorable outcomes that are expected to arise. (Arq Bras Endocrinol Metab 2007;51/2:275-280)
\end{abstract}

Keywords: Cardiovascular diseases; Diabetes mellitus; Mortality; Evidencebased medicine revisão

\author{
JUAREZ R. DE BRAGA \\ ÍtALO S.O. SANTOS \\ URI P. FLATO \\ HélIO P. Guimarães \\ Álvaro AVEzUM
}

Divisão de Pesquisa do Instituto

Dante Pazzanese de

Cardiologia, São Paulo, SP.
Recebido em 11/11/06

Aceito em 16/11/06 
$\mathrm{O}$ DIABETES MELLITUS (DM) representa a quinta causa de morte no mundo (1), o que ocorre principalmente em conseqüência das doenças cardiovasculares. Estima-se que ele eleve o risco de sua ocorrência, em especial o da doença arterial coronariana (DAC), em duas a quatro vezes (2), sendo o terceiro fator de risco mais importante na sua gênese (3).

Conseqüentemente, o DM aumenta também o risco de síndromes coronarianas agudas (SCA), cuja incidência chega a $20 \%$ em 7 anos, em diabéticos, contra 3,5\% em não-diabéticos - uma incidência semelhante àquela de indivíduos que já sofreram um infarto agudo do miocárdio (IAM) anteriormente (2). No Brasil, a presença de valores basais de glicemia $\geq 126 \mathrm{mg} / \mathrm{dL}$ oferece um risco quase 3 vezes maior para a ocorrência de infarto agudo do miocárdio (4). No contexto das SCA, o DM determina, ainda, um pior prognóstico, tanto a curto prazo, com uma mortalidade durante a internação que chega a ser duas vezes maior que a de não-diabéti$\cos (5)$, quanto a longo prazo, após a alta hospitalar $(6,7)$. Atualmente, sabe-se que não somente o diabetes, mas também a tolerância diminuída à glicose e demais estados hiperglicêmicos, determinam um aumento no risco de DAC e eventos coronarianos agudos $(8,9)$.

Infelizmente, apesar de todos os avanços na Cardiologia, em especial no tratamento das SCA, os diabéticos não têm experimentado uma diminuição significativa nas taxas de mortalidade como aquela experimentada por indivíduos não-diabéticos $(5,10)$.

Segundo estimativas, o número de diabéticos no mundo deve dobrar nas próximas décadas, totalizando 300 milhões de indivíduos no ano de 2030 (11); desses, 11 milhões só no Brasil. Tendo em vista esse aumento na prevalência do diabetes e demais estados hiperglicêmicos, o que se espera é um aumento correspon- dente na incidência das síndromes coronarianas agudas. Desta forma, torna-se fundamental entender o porquê do risco cardiovascular aumentado nesses pacientes, de modo a estabelecer quais as melhores estratégias para o seu adequado tratamento.

\section{FISIOPATOLOGIA DO RISCO CARDIOVASCULAR AUMENTADO EM DIABÉTICOS}

Trombose sobre uma placa de ateroma no território das artérias coronárias é o processo fisiopatológico subjacente a uma síndrome coronariana aguda. A trombose formada a seguir pode levar a uma oclusão do vaso que varia entre subtotal e total, determinando um quadro clínico que se apresenta entre a angina instável, infarto agudo do miocárdio (com e sem supradesnivelamento do segmento ST), e a morte súbita (12).

As síndromes coronarianas agudas ocorrem em conseqüência de um enfraquecimento focal da placa de ateroma, que sofre ruptura e subseqüente trombose (12). As placas suscetíveis à ruptura são aquelas com menor espessura da capa fibrosa e maior quantidade de lípides - as placas ditas instáveis, que são justamente o tipo mais freqüente em diabéticos (13). Essa predisposição à formação de placas instáveis no DM decorre do estado metabólico anormal vigente nesses pacientes, que leva a alterações no funcionamento de várias células: células endoteliais, células inflamatórias e células musculares lisas. Além disso, há uma tendência pronunciada à trombose, devido a alterações funcionais nas plaquetas e na cascata de coagulação (figura 1).

A disfunção das células endoteliais ocorre por mecanismos diversos, e tem como evento central a redução dos níveis de óxido nítrico. $\mathrm{O}$ óxido nítrico, na

Figura 1. Alterações induzidas pelo diabetes que aumentam o risco de doença arterial coronariana e síndrome coronariana aguda.

\begin{tabular}{|c|c|c|}
\hline ENDOTÉLIO & INFLAMAÇÃO & HIPERCOAGULABILIDADE \\
\hline 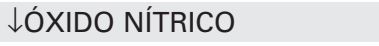 & 个RECEPTORES & 个FATOR VII \\
\hline 个ENDOTELINA-1 & 个CITOCINAS & 个FIBRINOGÊNIO \\
\hline 个ANGIOTENSINA-II & 个METALOPROTEINASES & $\downarrow$ ANTITROMBINA III \\
\hline \multirow[t]{2}{*}{ 个RADICAIS LIVRES DE O2 } & 个CÉLULAS INFLAMATÓRIAS & $\downarrow$ PROTEÍNA C \\
\hline & & $\downarrow \mathrm{PAI}-1$ \\
\hline CÉLULAS MUSCULARES & PLAQUETAS & \\
\hline \multirow[t]{4}{*}{ \SÍNTESE DE COLÁGENO } & 个RECEPTORES Gp & \\
\hline & 个TROMBOXANO A2 & \\
\hline & TDIMETRO & \\
\hline & ADESIVIDADE/AGREGAÇÃO & \\
\hline
\end{tabular}

Gp = glicoproteína PAI-1= inibidor da ativação do plasminogênio-1 
microcirculação, tem propriedades vasodilatadoras e inibe tanto a ativação plaquetária quanto à migração de células inflamatórias. Com a redução dos seus níveis, todas essas ações ficam comprometidas. Paralelamente, ocorre um aumento de substâncias vasoconstrictoras, como a endotelina- 1 e a angiotensina-II, bem como um aumento de radicais livres de oxigênio (14).

Ocorre, ainda, um aumento da expressão de receptores de adesão de células inflamatórias (14), que leva a um aumento do número de linfócitos $\mathrm{T}$ e monócitos nas placas de ateroma. Os linfócitos $\mathrm{T}$ estimulam a produção local de citocinas e metaloproteinases, que determinam uma diminuição na síntese dos componentes da capa fibrosa - um dos fatores que levam uma placa a se tornar instável. Já os monócitos, no espaço sub-endotelial, tornam-se macrófagos que irão ingerir partículas de lipoproteínas de baixa densidade (LDL) oxidadas, gerando as células espumosas, que determinam o conteúdo de lípides da placa outro fator determinante do grau de instabilidade (15). As células espumosas irão, ainda, sintetizar interleucinas que estimulam a aderência de mais células inflamatórias, gerando um círculo vicioso aterogênico (15). As células musculares lisas são estimuladas a sofrer apoptose, o que resulta em menor síntese de colágeno, levando ao enfraquecimento da capa fibrosa (15).

As plaquetas nos diabéticos têm, caracteristicamente, um diâmetro aumentado, uma maior quantidade de receptores de glicoproteína (Gp) IIb/IIIa e Ib em suas superfícies, sintetizam maiores quantidades de tromboxano A2 e exibem adesividade e agregação mais intensas. Tudo isso exacerba as conseqüências no caso de uma ruptura da placa, aumentando as chances de trombose (14).

Paralelamente, o DM induz um estado de hipercoagulabilidade, por aumentar a expressão do fator tecidual, fator VII e fibrinogênio, e diminuir o número de anticoagulantes endógenos, como a antitrombina III e a proteína C. Ocorre, ainda, uma redução na atividade do sistema fibrinolítico, pelo aumento nos níveis do inibidor da ativação do plasminogênio-l (PAI-1), o que favorece ainda mais uma trombogênese exacerbada nesses pacientes (15).

\section{CARACTERÍSTICAS CLÍNICAS DAS SIINDROMES CORONARIANAS AGUDAS EM DIABÉTICOS}

Pacientes com diabetes e SCA apresentam-se freqüentemente com sintomas e sinais atípicos, provavelmente devido à neuropatia sensitiva e autonômica. Nessa subpopulação, predominam dispnéia, sudorese, náuseas e vômitos em relação à dor torácica (16), o que leva a um retardo na procura por serviços de emergência e, conseqüentemente, posterga o início do seu tratamento (17). A interpretação de eletrocardiogramas também é mais difícil nesses pacientes, visto que as sulfoniluréias, usadas no tratamento da hiperglicemia, parecem diminuir o grau de alterações eletrocardiográficas, por uma diminuição da sensibilidade do método, reduzindo a amplitude de alterações do segmento ST e onda $\mathrm{T}(18)$.

Durante a internação, os diabéticos apresentam taxas mais elevadas de mortalidade que os não-diabéticos. Esses números podem chegar a $11,7 \%, 6,3 \%$ e $3,9 \%$, no caso de IAM com supradesnivelamento do segmento ST, IAM sem supradesnivelamento do segmento ST e angina instável, respectivamente, contra $6,4 \%, 5,1 \%$ e $2,9 \%$ em não-diabéticos (5). $\mathrm{O}$ diabetes, naqueles que sobrevivem à fase aguda de um evento coronariano, eleva a mortalidade ao longo do tempo em valores que variam entre 7 a $18 \%$ em 30 dias, 15 a $34 \%$ após 1 ano e até $43 \%$ após 5 anos (17-19,21). O risco relativo para mortalidade após uma SCA em diabéticos varia entre 1,3 e 5,4, após ajustes estatísticos para fatores de confusão (12) (tabela l).

Vários fatores têm sido implicados na determinação de um pior prognóstico nos diabéticos que tiveram uma SCA: a extensão da disfunção ventricular, a persistência de isquemia no miocárdio residual, as alterações pró-trombóticas, e o desequilíbrio no sistema nervoso autonômico, que predispõe a arritmias (6). Esses fatores levam a um risco de morte aumentado, gerando principalmente reinfarto e insuficiência cardíaca. Além desses, a instabilidade elétrica (arritmias ventriculares, bloqueios atrioventriculares e morte súbita), o acidente vascular cerebral e o choque cardiogênico também contribuem para uma maior mortalidade nessa população. O reinfarto, que ocorre devido a uma DAC difusa, associada a alterações pró-trombóticas, tem uma chance de incidência de até 1,8 vezes após SCA em diabéticos (21).

$\mathrm{Na}$ internação, assim como a longo prazo, a insuficiência cardíaca é a complicação mais comum. Sua incidência durante a internação varia entre $15 \%$, em caso de angina instável, e 30\%, no caso de IAM. Esses valores correspondem praticamente ao dobro da incidência em pacientes não-diabéticos (5). Após a alta, a incidência de insuficiência cardíaca ainda permanece elevada, chegando a 1,3\% ao ano (12). Estimase que isso possa ser devido a uma doença aterosclerótica mais difusa - que pode influenciar negativamente o processo de remodelação após um IAM ou, ainda, a uma miocardiopatia gerada pelo aumento dos triglicérides sangüíneos, alteração metabólica que freqüentemente acompanha o diabetes (6). 
Tabela 1. Estudos de sobrevivência após evento coronariano agudo - comparação de desfechos entre diabéticos e não-diabéticos.

\begin{tabular}{lccccccc}
\hline ESTUDO & TIPO & SEGUIMENTO & n (n diabetes) & DIAGNÓSTICO & $\begin{array}{c}\text { MORTALIDADE } \\
\text { DIM x NÃO-DM (\%) }\end{array}$ & RR (IC 95\%) \\
& & & & & & \\
GRACE (9) & REGISTRO & HOSPITALAR & $16.116(16.116)$ & IAM com SST & $11,7 \times 6,4$ & $1,48(1,0-2,1)$ \\
& & & & IAM sem SST & $6,3 \times 5,1$ & $1,14(0,8-1,5)$ \\
& & & & Al & $3,9 \times 2,9$ & $1,41(1,0-1,9)$ \\
OASIS (19) & REGISTRO & 2 anos & $8.013(1.718)$ & SCA sem ST & $18 \times 10$ & $1,57(1,3-1,8)$ \\
FRISC-II (20) & ERC & 2 anos & $2.457(299)$ & SCA sem ST & $12,5 \times 2,7$ & $5,4(2,1-14)$ \\
GUSTO-I (17) & ERC & 1 ano & $41.021(5.944)$ & IAM com SST & $14,5 \times 8,9$ & $1,7(-)$ \\
GUSTO-IIB (21) & ERC & 30 dias & $12.142(2.175)$ & IAM com SST & $8,4 \times 5,5$ & $1,5(1,2-2,1)$ \\
& & & & SCA sem ST & $6,2 \times 3,3$ & $1,9(1,5-2,5)$ \\
\hline
\end{tabular}

$\mathrm{n}=$ número total de pacientes incluídos no estudo; $\mathrm{n}$ diabetes= número de pacientes diabéticos incluídos no estudo; $\mathrm{ERC}=$ estudo randomizado controlado; IAM com SST= infarto agudo do miocárdio com supra-desnivelamento do segmento ST; IAM sem SST = infarto agudo do miocárdio sem supra-desnivelamento do segmento ST; SCA sem SST= síndrome coronariana aguda sem supra-desnivelamento do segmento ST; RR= risco relativo; IC= intervalo de confiança.

\section{INTERVENÇÕES}

Sendo o risco absoluto de mortalidade maior em diabéticos quando comparado ao de não-diabéticos, as intervenções nessa população trazem maior impacto quanto aos benefícios $(18,22)$. Manejar agressivamente a hiperglicemia intra-hospitalar com insulina parece reduzir a mortalidade a longo prazo (23), bem como evitar a hipoglicemia (24). No entanto, ainda existem uma série de questionamentos a esse respeito (25-27). Em pacientes com infarto agudo do miocárdio com supradesnivelamento do segmento $S T$, a terapia de reperfusão, seja por trombólise, seja por intervenção coronariana percutânea, traz mais benefícios em diabéticos do que em não-diabéticos. A trombólise traz uma redução absoluta da mortalidade duas vezes maior que em não-diabéticos, salvando 35 vidas por 1.000 doentes trombolisados quando comparadas a 15 em não-diabéticos (28).

$\mathrm{Na}$ intervenção coronariana percutânea, os benefícios parecem ser semelhantes em diabéticos e não-diabéticos, porém com taxas menores de eventos adversos em 30 dias nos diabéticos (18). No infarto agudo do miocárdio sem supradesnivelamento do segmento ST e angina instável, tanto diabéticos como não-diabéticos se beneficiam de uma estratégia invasiva precoce (estudo angiográfico em até 48 horas durante a internação e revascularização se necessário) (29). Quando adotada essa estratégia, no entanto, os benefícios são maiores em diabéticos do que em não diabéticos $(20,30)$.

Inibidores da glicoproteína IIb/IIIA (iGPIIb/ IIIA) mostraram que sua administração reduz a freqüência de morte, reinfarto e isquemia recorrente em 30 dias, quando usados como terapia primária em sín- dromes coronarianas sem supra de ST. Os benefícios dos iGPIIb/IIIA aparecem especialmente quando usados em indivíduos com marcadores de alto risco, como alterações dinâmicas do segmento ST, marcadores de necrose positivos e o diabetes mellitus (31).

O uso combinado de AAS e clopidogrel em pacientes com síndrome coronariana aguda sem supradesnivelamento do segmento ST não mostrou diferenças no benefício entre diabéticos e não-diabéti$\cos (32)$. No entanto, em uma sub-análise do estudo, benefícios maiores foram trazidos para a subpopulação submetida à intervenção coronariana percutânea (18).

Beta-bloqueadores têm eficácia comprovada após um IAM, reduzindo as taxas de reinfarto e mortalidade. Em diabéticos, parece haver um efeito similar ou um leve benefício quando comparado ao de nãodiabéticos $(33,34)$.

Inibidores da ECA já demonstraram, em inúmeros estudos randomizados, a importância na diminuição da morbimortalidade após a sua introdução nas fases iniciais do infarto agudo do miocárdio (35). Entre os pacientes com diabetes, os benefícios foram maiores que entre não-diabéticos: 17,3 vidas salvas por 1.000 pacientes diabéticos tratados, contra 3,2 vidas salvas por 1.000 pacientes não-diabéticos tratados. Apesar de não estudados de maneira específica no contexto das SCA sem supra de ST, provavelmente têm benefício nesses pacientes também, especialmente em quadros clínicos com outros fatores de risco ou outras manifestações de doença cardiovascular.

Algumas intervenções têm eficácia comprovada no tratamento das SCA, mas ainda não dispõem de evidências especificamente relacionadas à população diabética. É o caso, por exemplo, das estatinas, que, quan- 
do administradas de maneira precoce (em menos de 4 semanas) após uma SCA, reduzem marcadores inflamatórios e a incidência de isquemia recorrente $(36,37)$. O seu emprego em altas doses reduz, ainda, novos eventos coronarianos e mortalidade (38). Outros exemplos seriam os antitrombínicos, e o clopidogrel, que, quando usados no IAM com supradesnivelamento do segmento $\mathrm{ST}$, associados à trombólise ou à intervenção coronariana percutânea, resultam em melhores taxas de reperfusão (39). Entretanto, ainda não existem evidências de que os seus benefícios sejam superiores em diabéticos.

\section{LACUNAS ENTRE AS EVIDÊNCIAS E A PRÁTICA CLÍNICA}

Apesar dos benefícios das intervenções baseadas em evidências no tratamento das SCA em diabéticos, chama atenção a sub-utilização dessas medidas, o que também contribui para a maior morbimortalidade dessa população (40). Conforme dados obtidos do estudo CRUSADE, registro americano de pacientes com SCA sem supra de ST, os pacientes diabéticos tiveram menor chance de serem tratados com antiplaquetários, antitrombínicos e beta-bloqueadores. Tiveram, ainda, menor chance de serem submetidos à coronariografia ou intervenção coronariana percutânea, especialmente dentro de 48 horas após a internação. Quando se usou método estatístico para controlar os fatores que seriam potenciais contra-indicações para as terapias, como a insuficiência renal crônica, diferenças significativas ainda permaneceram (4l). Quando considerados os pacientes com infarto agudo do miocárdio, diabéticos tiveram menor chance de receberem AAS, beta-bloqueadores e serem submetidos a procedimento de revascularização $(7,40)$. São, ainda, menos propensos a receber trombolítico (42), com uma chance $50 \%$ menor que não-diabéticos (43). A utilização de intervenção coronariana percutânea mostra as mesmas tendências que no caso da fibrinólise - tem menor chance de ser usada em diabéticos (44). $\mathrm{Na}$ alta, os pacientes diabéticos tendem a receber menos medicações para prevenção secundária. $\mathrm{O}$ uso de antiagregantes plaquetários, estatinas, inibidores da ECA e beta-bloqueadores é menor que em não-diabéticos que sofreram IAM (45).

\section{CONCLUSÕES}

Tendo em vista o aumento da prevalência do diabetes mellitus previsto para os próximos anos e levando-se em conta que as síndromes coronarianas agudas serão a principal causa de mortalidade nessa população, tornase cada vez mais necessário que cardiologistas e endocrinologistas passem a interagir, de maneira a modificar o panorama previsto. Para tanto, devem se valer de maneira agressiva das evidências já estabelecidas, buscando, além de um controle glicêmico rigoroso e dos demais fatores de risco cardiovascular, o adequado tratamento das síndromes coronarianas agudas.

\section{REFERÊNCIAS}

1. Roglic G, Unwin N, Bennett P, Mathers C, Tuomilehto J, Nag $S$, et al. The burden of mortality attributable to diabetes: realistic estimates for the year 2000. Diabetes Care 2005;28:2130-5.

2. Haffner SM, Lehto S, Rönnemaa T, Pyörälä K, Laakso M. Mortality from coronary heart disease in subjects with type 2 diabetes and in nondiabetic subjects with and without prior myocardial infarction. N Engl J Med 1998;339(4):229-34.

3. Yusuf S, Hawken S, Ôunpuu S, Dans T, Avezum A, Lanas F, et al. Effect of potentially modifiable risk factors associated with myocardial infarction in 52 countries (the INTERHEART study): case-control study. Lancet 2004;364:937-52.

4. Piegas LS, Avezum A, Pereira JCR, Rossi Neto JM, Hoepfner C, Farran JA, et al.; on behalf of the AFIRMAR study investigators. Risk factors for myocardial infarction in Brazil. Am Heart J 2003; 146:331-8.

5. Franklin K, Goldberg RJ, Spencer F, Klein W, Budaj A, Brieger D. Implications of diabetes in patients with acute coronary syndromes. The global registry of acute coronary events. Arch Intern Med 2004; 164:1457-63.

6. Aronson D, Rayfield EJ, Chesebro JH. Mechanisms determining course and outcome of diabetic patients who have had acute myocardial infarction. Ann Intern Med 1997;126:296306.

7. Berger AK, Breall JA, Gersh BJ, Johnson AE, Oetgen WJ, Marciniak TA, et al. Effect of diabetes mellitus and insulin use on survival after acute myocardial infarction in the elderly (The Cooperative Cardiovascular Project). Am J Cardiol 2001;87:272-7.

8. Brunner EJ, Shipley MJ, Witte DR, Fuller JH, Marmot MG. Relation between blood glucose and coronary mortality over 33 years in the Whitehall Study. Diabetes Care 2006;29:26-31.

9. Coutinho M, Gerstein HC, Wang Y, Yusuf S. The relationship between glucose and incident cardiovascular events. A meta regression analysis of published data from 20 studies of 95,783 individuals followed for 12.4 years. Diabetes Care 1999;22:233-40.

10. Gu K, Cowie CC, Harris MI. Diabetes and decline in heart disease mortality in US adults. JAMA 1999;281:1291-7.

11. Wild S, Roglic G, Green A, Sicree R, King H. Global prevalence of diabetes: estimates for 2030. Diabetes Care 2004:27:1047-53.

12. Bartnik M, Malmberg K, Rydén L. Management of patients with type 2 diabetes after acute coronary syndromes. Diabetes Vasc Dis Res 2005;2:144-54.

13. Moreno PR, Murcia AM, Palacios IF. Coronary composition and macrophage infiltration in atherectomy specimens from patients with diabetes mellitus. Circulation 2000;102:2180-4.

14. Beckman JA, Creager MA, Libby P. Diabetes and atherosclerosis - Epidemiology, pathophysiology and management. JAMA 2002;287:2570-81.

15. Carter AM. Inflammation, thrombosis and acute coronary syndromes. Diabetes Vasc Dis Res 2005;2:113-21.

16. Marchant B, Umachandran V, Stevenson R, Kopelman PG, Timmis AD. Silent myocardial ischemia: role of subclinical neuropathy in patients with and without diabetes. J Am Coll Cardiol 1993;22:1433-7. 
17. Mak KH, Moliterno DJ, Granger CB, Miller DP, White HD, Wilcox RG, et al. Influence of diabetes mellitus on clinical outcome in the thrombolytic era of acute myocardial infarction. GUSTO-I Investigators. Global Utilization of Streptokinase and Tissue plasminogen activator for Occluded coronary arteries. J Am Coll Cardiol 1997;30:171-9.

18. Trichon $\mathrm{BH}$, Roe MT. Acute coronary syndromes and diabetes mellitus. Diabetes Vascul Dis Res 2004;1:23-32.

19. Malmberg K, Yusuf S, Gerstein HC, Brown J, Zhao F, Hunt D, et al. Impact of diabetes on long-term prognosis in patients with unstable angina and non-O-wave myocardial infarction: results of the OASIS (Organization to Assess Strategies for Ischemic Syndromes) Registry. Circulation 2000;102:1014-9.

20. FRagmin and Fast Revascularisation during Instability in Coronary artery disease (FRISC II) Investigators. Invasive compared with non-invasive treatment in unstable coronaryartery disease: FRISC II prospective randomised multicentre study. Lancet 1999;354:708-15.

21. McGuire DK, Emanuelsson $\mathrm{H}$, Granger CB, Magnus Ohman E, Moliterno DJ, White HD, et al. Influence of Diabetes mellitus on clinical outcomes across the spectrum of acute coronary syndromes: findings from the GUSTO-IIb study. Eur Heart J 2000:21:1750-8.

22. Hildebrandt P. Diabetic patients and acute coronary syndromes. Eur Heart J 2001;22:887-8.

23. DIGAMI-I Randomized trial of insulin-glucose infusion followed by subcutaneous insulin treatment in diabetic patients with acute myocardial infarction (DIGAMI study): effects on mortality at 1 year. J Am Coll Cardiol 1995;26(1):57-65.

24. Svensson AM, McGuire DK, Abrahamsson P, Dellborg M. Association between hyper- and hypoglycemia and 2-year all-cause mortality risk in diabetic patients with acute coronary events. Eur Heart J 2005;26:1255-61.

25. Malmberg K, Ryden L, Wedel H, Birkeland K, Bootsma A, Dickstein $\mathrm{K}$, et al, for the DIGAMI-II investigators. DIGAMI-II Intense metabolic control by means of insulin in patients with diabetes mellitus and acute myocardial infarction (DIGAMI 2): effects on mortality and morbidity. Eur Heart J 2005;26(7):650-61.

26. The CREATE-ECLA Trial Group Investigators. Effect of glucose-insulinpotassium infusion on mortality in patients with acute ST-segment elevation myocardial infarction. JAMA 2005;293:437-46.

27. van der Horst IC, Zijlstra F, van't Hof AW, Doggen CJ, de Boer MJ, Suryapranata H, et al.; Zwolle Infarct Study Group. Glucose-insulin-potassium infusion in patients treated with primary angioplasty for acute myocardial infarction: the glucose insulin potassium study: a randomized trial. J Am Coll Cardiol 2003:42:784-91.

28. Fibrinolytic Therapy Trialists' (FTT) Collaborative Group. Indications for fibrinolytic therapy in suspected acute myocardial infarction: collaborative overview of early mortality and major morbidity results from all randomised trials of more than 1000 patients. Lancet 1994:343:311-22.

29. Mehta SR, Cannon CP, Fox KAA, Wallentin L, Boden WE, Spacek $R$, et al. Routine vs. selective invasive strategies in patients with acute coronary syndromes a collaborative metaanalysis of randomized trials. JAMA 2005;293(23):2908-17.

30. Cannon CP, Weintraub WS, Demopoulos LA, Vicari R, Frey MJ, Lakkis N, et al.; TACTICS (Treat Angina with Aggrastat and Determine Cost of Therapy with an Invasive or Conservative Strategy) - Thrombolysis in Myocardial Infarction 18 Investigators. Comparison of early invasive and conservative strategies in patients with unstable coronary syndromes treated with the glycoprotein Ilb/llla inhibitor tirofiban. N Engl J Med 2001;344(25):1879-87.

31. Roffi M, Chew DP, Mukherjee D, Bhatt DL, White JA, Heeschen C, et al. Platelet glycoprotein Ilb/llla inhibitors reduce mortality in diabetic patients with non-ST-segmentelevation acute coronary syndromes. Circulation 2001; 104:2767-71.

32. Yusuf S, Zhao F, Mehta SR, Chrolavicius S, Tognoni G, Fox KK; Clopidogrel in Unstable Angina to Prevent Recurrent Events Trial Investigators. Effects of clopidogrel in addition to aspirin in patients with acute coronary syndromes without ST-segment elevation. N Engl J Med 2001;345:494-502.
33. Malmberg K, Herlltz J, Hjalmarson A, Ryden L. Effects of metoprolol on mortality and late infarction in diabetics with suspected acute myocardial infarction. Retrospective data from two large studies. Eur Heart J 1989;10:423-8.

34. Kjekshus J, Gilpin E, Cali G, Blackey AR, Henning H, Ross J Jr. Diabetic patients and $\beta$-blockers after acute myocardial infarction. Eur Heart J 1990;11:43-50.

35. ACE Inhibitor Myocardial Infarction Collaborative Group. Indications for ACE inhibitors in the early treatment of acute myocardial infarction: systematic overview of individual data from 100,000 patients in randomized trials. ACE Inhibitor Myocardial Infarction Collaborative Group. Circulation 1998; $97: 2202-12$.

36. Schwartz GG, Olsson AG, Ezekowitz MD, Ganz P, Oliver MF, Waters D, et al.; Myocardial Ischemia Reduction with Aggressive Cholesterol Lowering (MIRACL) Study Investigators. Effects of atorvastatin on early recurrent ischemic events in acute coronary syndromes: the MIRACL study: a randomized controlled trial. JAMA 2001;285:1711-8.

37. Kinlay S, Schwartz GG, Olsson AG, Rifai N, Leslie SJ, Sasiela WJ, et al.; Myocardial Ischemia Reduction with Aggressive Cholesterol Lowering Study Investigators. High-dose atorvastatin enhances the decline in inflammatory markers in patients with acute coronary syndromes in the MIRACL study. Circulation 2003:108:1560-6.

38. Cannon $\mathrm{CP}$, Braunwald E, McCabe CH, Rader DJ, Rouleau JL, Belder R, et al.; Pravastatin or Atorvastatin Evaluation and Infection Therapy-Thrombolysis in Myocardial Infarction 22 Investigators. Intensive versus moderate lipid lowering with statins after acute coronary syndromes. N Engl J Med 2004:350:1495-504.

39. Sabatine MS, Cannon CP, Gibson CM, Lopez-Sendon JL, Montalescot G, Theroux P, et al.; CLARITY-TIMI 28 Investigators. Addition of clopidogrel to aspirin and fibrinolytic therapy for myocardial infarction with ST-segment elevation. $\mathbf{N}$ Engl J Med 2005;352:1179-89.

40. Norhammara A, Malmberga K, Rydena L, Tornvalla P, Stenestrandb $U$, Wallentinc $L$, for the Register of Information and Knowledge about Swedish Heart Intensive Care Admissions (RIKS-HIA). Under utilisation of evidence-based treatment partially explains for the unfavourable prognosis in diabetic patients with acute myocardial infarction. Eur Heart J 2003; $24: 838-44$

41. Brogan GX, Peterson ED, Mulgund J, Bhatt D, Ohman EM, Gibler WB, et al. Treatment disparities in the care of patients with and without diabetes presenting with non-ST-segment elevation acute coronary syndromes. Diabetes Care 2006;29:9-14.

42. Pfeffer MA, Moye LA, Braunwald E, Basta L, Brown EJ Jr, Cuddy TE, et al. Selection bias in the use of thrombolytic therapy in acute myocardial infarction. The SAVE Investigators. JAMA 1991;266:528-32.

43. Barron HV, Bowlby LJ, Breen T, Rogers WJ, Canto JG, Zhang $Y$, et al. Use of reperfusion therapy for acute myocardial infarction in the United States: data from the National Registry of Myocardial Infarction 2. Circulation 1998;97:1150-6.

44. Collet JP, Montalescot G. The acute reperfusion management of STEMI in patients with impaired glucose tolerance and type 2 diabetes Diabetes Vascul Dis Res 2005;2(3):136-43.

45. Chowdhury TA, Lasker SS, Dyer PH. Comparison of secondary prevention measures after myocardial infarction in subjects with and without diabetes mellitus. J Int Med 1999;245:565-70.

Endereço para correspondência:

Álvaro Avezum

Avenida Dr. Dante Pazzanese 500, $1^{\circ}$ andar

04012-909 São Paulo, SP

E-mail: avezum@yahoo.com 